\title{
Ultrasonic signal processing using wavelet transform for automatic rail defect detection
}

\author{
Gang $\mathrm{Yu}^{\mathrm{a}}$, Xiaoman Wang ${ }^{\mathrm{b}}$ \\ Harbin Institute of Technology (HIT) Shenzhen Graduate School, Shenzhen, Guangdong \\ 518055, P.R.China \\ a969083604@qq.com, b15245035653@163.com
}

Keywords: Ultrasonic, rail defect detection, wavelet transform, denoising.

Abstract. Ultrasonic echo signal from running rail defect detection equipment generally contains noise, which makes it hard to identify the defects. In this paper, wavelet transform based approach is proposed to process the ultrasonic echo signal. Through the experimental verification, the proposed method is effective in detecting the defects of rail under running operation.

\section{Introduction}

Traditionally, ultrasonic is used to detect the defects of the rail, and it mainly depends on experienced or specially trained workers who observe the ultrasonic echo signal to judge whether the rail has defects. In the process of detection, because of the ultrasonic probe moving on the rail, the defect echo signal is disturbed, and even submerged by the noise or other interferences. Workers can't identify the defects from the dynamic echo signal effectively $[1,2]$. In this paper, the ultrasonic echo signal is decomposed and reconstructed using wavelet transform for denoising, which make the defect signal more easy to be identified.

\section{The Construction of the Nondestructive Defect Detection Experiment Equipment}

At present, domestic nondestructive defect detection devices have low intelligence, low detection rate of the rail defects. In order to solve these problems, this paper designs a new type of monorail defect detection experiment in order to improve the current technology as shown in Figure 1. Ultrasonic probes contact with the rail directly using water as coupling agent. The ultrasonic probes of the whole device mainly have four parts as shown in Table 1. Probes at different angles are used to detect different defects, such as $70^{\circ}$ probe detecting rail head defect, $37^{\circ}$ probe detecting threaded hole defects and $0^{\circ}$ probe detecting the defects of rail surface to the bottom of the rail and the waist projection parts [3]. In Figure 2, we can see the $0^{\circ}$ probe is focused to detecting two hole defects.

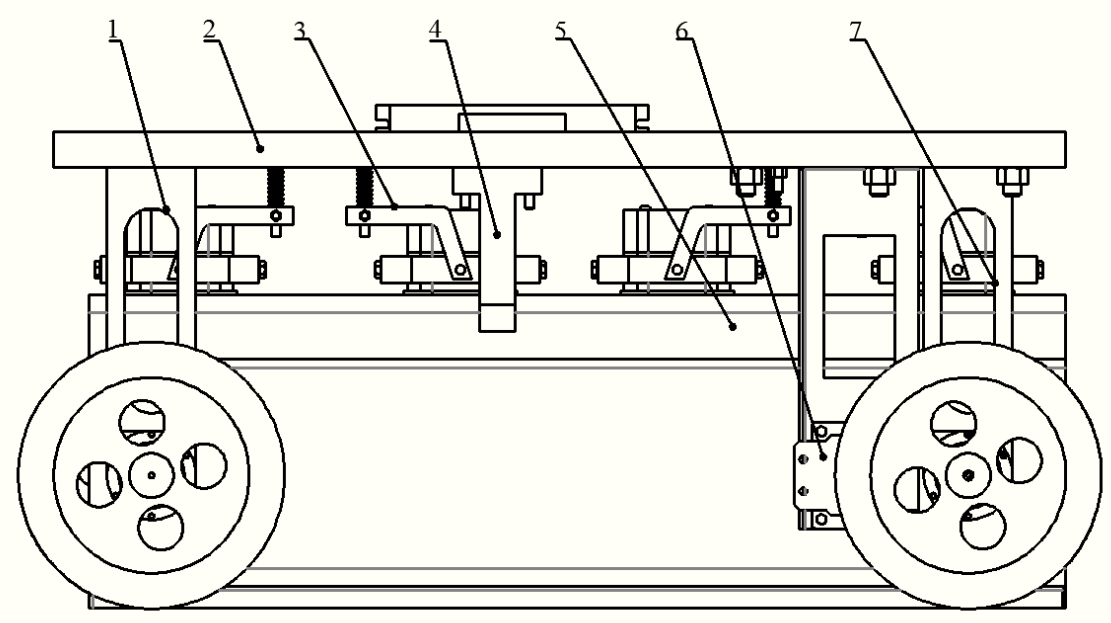

Figure1 Monorail nondestructive defect detection experiment device.

1. The driven system. 2. Baseplate. 3. Defect detection system.

4. Guide structure. 5. Rail. 6. The motor. 7. Drive system. 
Table 1 The types of probes in detection device

\begin{tabular}{ccc}
\hline The serial number & The probe model & angle of avertence \\
\hline 1 & $0^{\circ}+37^{\circ}$ & $0^{\circ}$ \\
2 & $37^{\circ}+70^{\circ}$ & $20^{\circ}$ \\
3 & $70^{\circ}+70^{\circ}$ & $20^{\circ}$ \\
4 & $70^{\circ}+70^{\circ}$ & $0^{\circ}$ \\
\hline
\end{tabular}
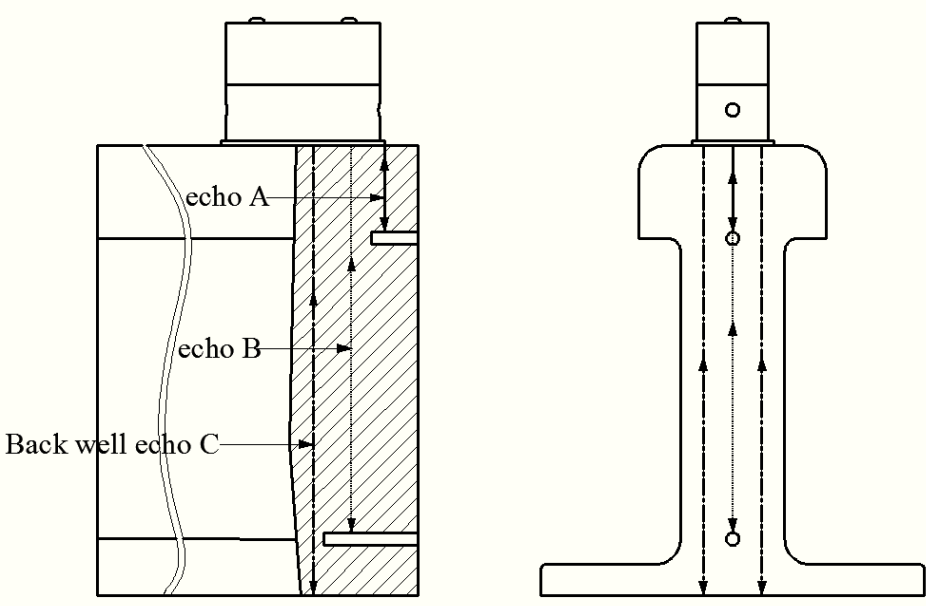

Figure. 2 The $0^{\circ}$ probes detect two holes flaws.

\section{The Defect Echo Signal Processing}

This experiment adopts the CTS - O4PC multichannel high speed data acquisition card, and the sampling frequency is $100 \mathrm{MHZ}$. Defect signal recognition mainly includes three steps. Firstly, the process of wavelet analysis is regarded as a decomposition function. The function is decomposed into wavelet coefficients [4]. Secondly, a soft threshold processing is adopted for the detail coefficients [5]. Finally, the original signal is recovered from the processing coefficients by wavelet reconstruction. In this paper, we take the situation in Figure 2 for instance as the defect echo.

Wavelet Decomposition. Wavelet transform is used for local analysis, which is to analyze a localized area of a larger signal. By the scaled and shifted operation, the approximations are the high-scale, low-frequency components of the signal and the details are the low-scale, high-frequency components, which can automatically adapt to the requirements of time-frequency signal analysis [6].The original signal passes through two complementary filters and emerges as two signals, which respectively are the approximations (low-pass filtering) and the details (high-pass filtering). Two signals are downsampled under the filter in the sampling of the scale of 2 [7]. Further decomposition means that the approximate signal as the original signal is decomposed according to the steps above.

Signal Denoising. In the non-destructive testing, due to data collection and environmental instruments, there are inevitably some independent noise components in echo signals. If the noise is not processed and analyzed directly, it is likely to make the results inaccurate. This paper uses the threshold denoising to process echo signal.

Assuming that the original signal is $\mathrm{f}(\mathrm{n})$, and $\mathrm{s}(\mathrm{n})$ is added the noise signal. So the basic model of the noise can be represented as:

$s(n)=f(n)+\sigma e(n)$

In the formula (1), e(n) is noise, and $\sigma$ is noise intensity. In the simplest case, e (n) is a Gaussian white noise, so $\sigma=1$. 
In the process of denoising, the key step is that how to assign a threshold for the coefficient, which directly affects the quality of the noise reduction. So people set up the model based on various theories and experience. However, no models are universal, and they all have their own range of applications.

From the theoretical model, this quantity is expressed by the formula (1). There are many methods to extract the $\sigma$ from $s(n)$. Assuming that the noise is white noise (the noise of the mathematical expectation is 0 ), $\sigma$ can be measured by standard deviation of wavelet decomposition coefficient in each layer of the original signal.

After getting noise intensity of the signal, according to the noise intensity $\sigma$, the threshold of each layer can be determined. In this paper the threshold can be decided by the Birge - Massart mathematical model.

Experimental Verification The experiment uses $0^{\circ}$ ultrasonic probe to detect two hole defects as shown in Figure 2. If the rail has defects, there are two reflections (echo signal A and echo signal B) by the two holes and a bottom echo signal C. If there are no defects in rail, there is only bottom echo signal C.

We use the $0^{\circ}$ probe of the flaw detection experiment device to get two groups of data (the static measurement data): normal signal (Figure 3 (a1)) and hole defect signal (Figure 3 (b1)). The two groups of signal can be processing respectively by wavelet decomposing, denoising and reconstructing. The signals are shown respectively in Figure 3: (a2) and (b2). Comparing with (b1) in Figure 3, (b2) is easier to recognize the defects. From (b2), we can find that there are two defects in the defect signal, respectively at $x=36.37 \mathrm{~mm}$ and $x=167.5 \mathrm{~mm}$, which associate with the actual defect location in the testing calibration block.
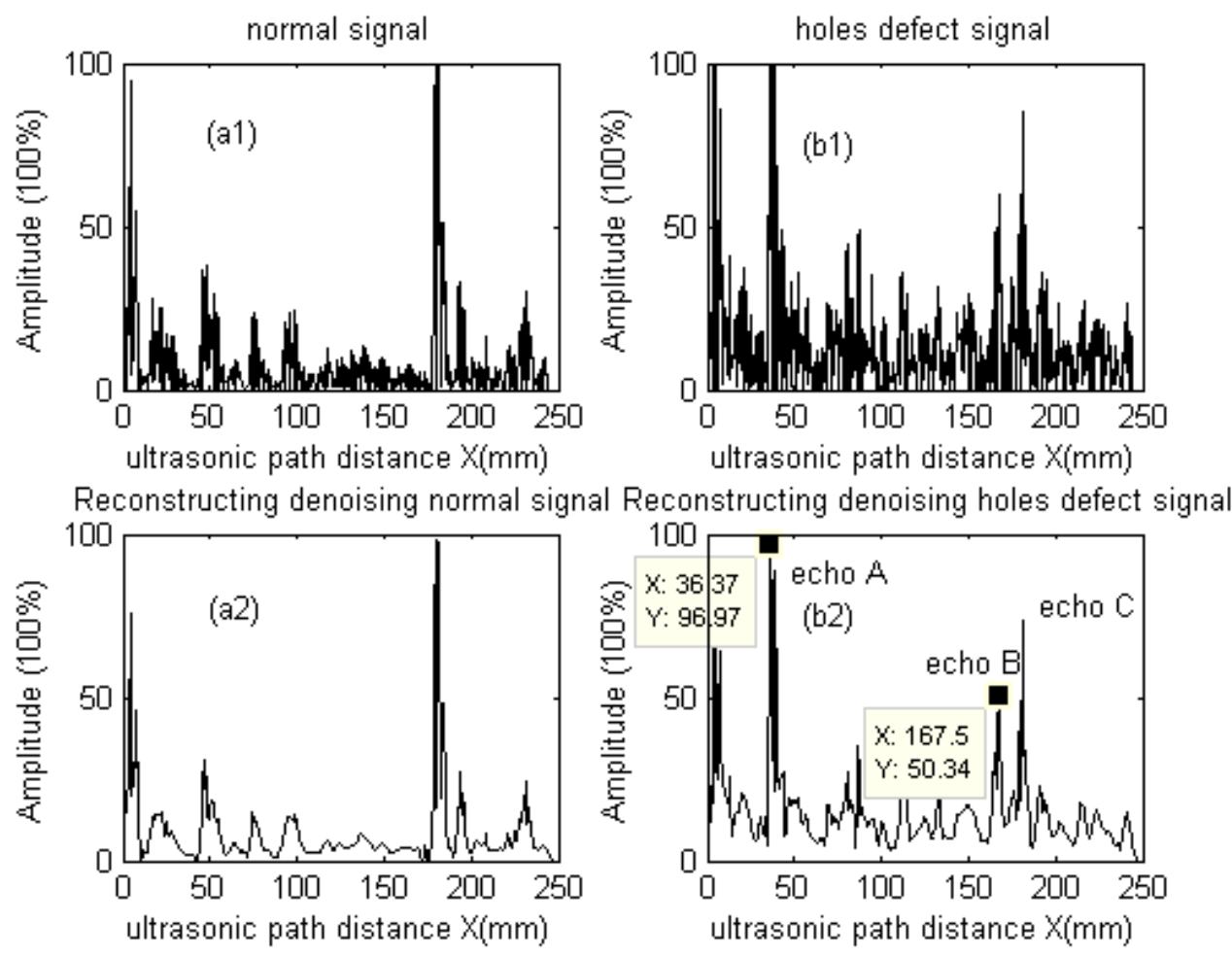

Figure 3 (a1) normal signal; (b1) holes defect signal; (a2) denoised normal signal; (b2) denoised hole defect signal.

In order to verify whether the wavelet processing meet the requirements in the dynamic state based on the probe velocity in $0.5 \mathrm{~m} / \mathrm{s}$, two sets of data (original signal in Figure 4(e1) and original signal in Figure 4(e2)) about the appearing to disappearing of defects are taken. At the same time, the experiment is used to detect defects in Figure 2. It is not hard to find that processing dynamic defect signal in Figure 4 (f1) is same as the static processing defect signal in Figure 3 (b2). After two groups of dynamic signal are processed, it is easy to see that the defect vary in two states, which is significantly 
and disappearing like Figure 4. Comparing the original signal and processing signals in Figure 4, the processing signal is easier to identify defects in dynamic state.
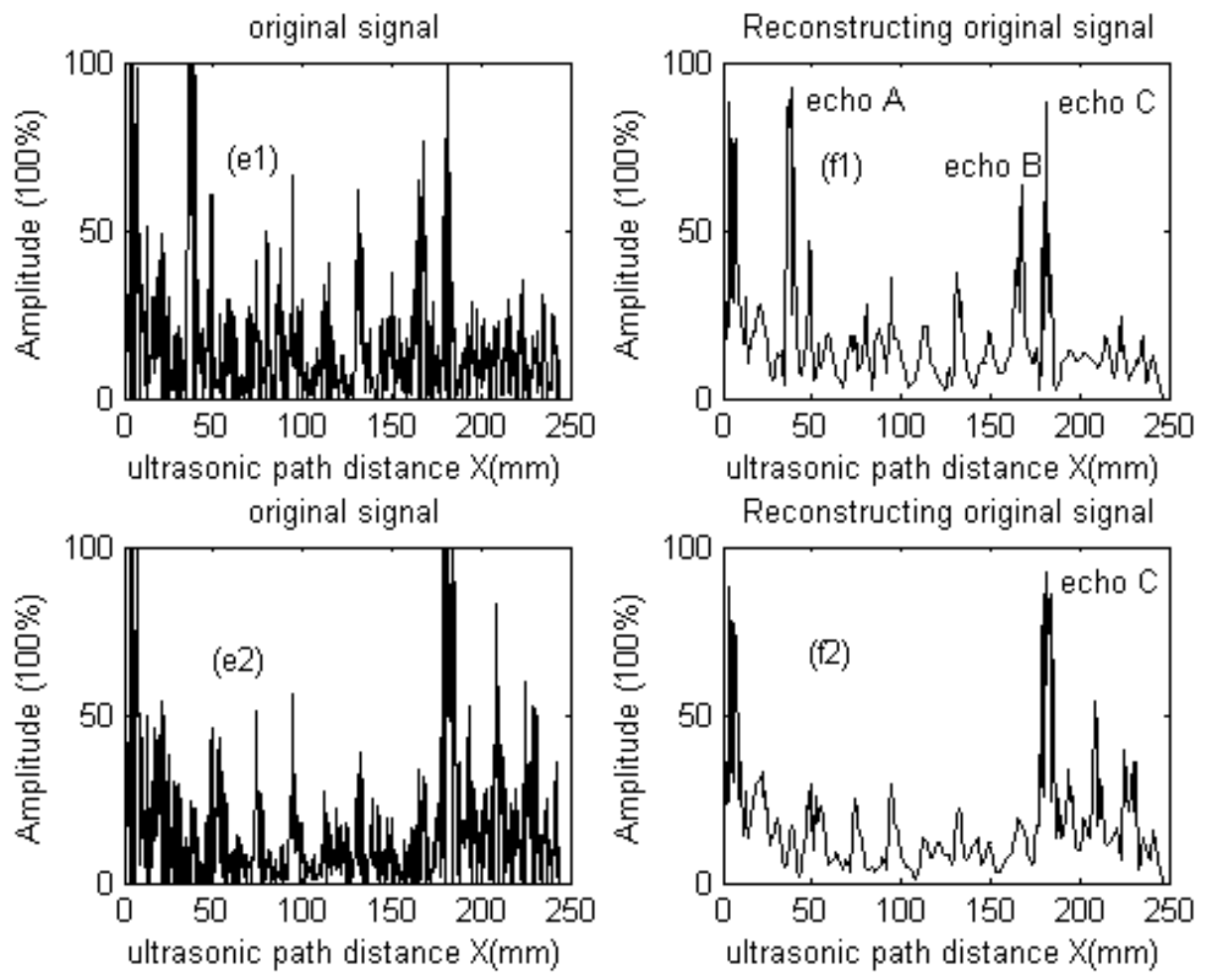

Figure 4 (e1) hole defects in original signal; (f1) denoised hole defects in original signal; (e2) normal original signal; (f2) denoised normal original signal.

\section{Conclusions}

In this paper, a wavelet based ultrasonic signal processing method is proposed for automatic rail defect detection. An experimental detection equipment is constructed for the verification of proposed approach, results have shown that it is effective for rail defect detection under both static and dynamic operation.

\section{References}

[1] Mcnamara J, Scalea F L D, Mcnamara J, et al. Improvements in Non-Contact Ultrasonic Testing of Rails by the Discrete Wavelet Transform [J]. Materials Evaluation, 2004, 62(3):365-372.

[2] Zhang Guangming, Ma Hongwei, Wang Yuwen. Ultrasonic nondestructive testing of flaw identification and noise suppression [J]. China mechanical engineering, 1999, 10 (12) : 1389-1391.

[3] Chen Chunsheng. Rail flaw detection work [M]. China railway publishing house, 2004

[4] Goswami J C, Chan A K. Fundamentals of wavelets: theory, algorithms, and applications [M]. Wiley, 2011.

[5] Donoho B D L. De-noising by soft-tresholding[C]// IEEE Trans. Inform. Theory. 1995.

[6] Cohen A, Ryan R D. Wavelets and multiscale signal processing [J]. English Translation Representations of the Cuntz Algebra, 1996.

[7] Lanza F, Rizzo P, Coccia S, et al. Non-contact ultrasonic inspection of rails and signal processing for automatic defect detection and classification[J]. Or Insight, 2005, 47(6):346-353. 